\title{
A Green's Function for Tectonomagnetic Problems in an Elastic Half-Space*
}

\author{
Yoichi SASAI \\ Earthquake Research Institute, University of Tokyo, Yayoi, Bunkyo-ku, Tokyo, Japan
}

(Received February 25, 1986; Revised April 25, 1986)

\begin{abstract}
A variety of tectonic models can be constructed by superposing the displacement field solution of a single force operative at a point in an elastic half-space. Piezomagnetic changes associated with the same models are given as well by the linear combination of fundamental piezomagnetic potentials, which arise from stressinduced magnetization produced by a unit single force at a point. The earth is assumed a homogeneous and isotropic elastic semi-infinite medium having a uniformly magnetized top layer. The method is adaptable to (a) surface load, (b) strain nuclei, (c) dislocation and (d) volume source problems. The prescribed potentials are obtained with the aid of a surface integral representation of the tectonomagnetic field (SASAI, 1983). As an application example, piezomagnetic field is calculated for a uniform circular load.
\end{abstract}

\section{Introduction}

Study of magnetic changes associated with earthquakes, volcanic activities and some other tectonic events is called "Tectonomagnetism" (NAGATA, 1969). The piezomagnetic effect of rocks under mechanical stresses is regarded as the principal cause of such phenomena (NAGATA, 1970; STACEY and JOHNSTON, 1972). The seismomagnetic effect (STACEY, 1964) has been our major interest with reference to the earthquake prediction research. Recent advances in measurement techniques enable us to detect some significant magnetic changes accompanying and/or preceding earthquakes (e.g., RIKITAKE, 1968; SMITH and JOHNSTON, 1976; OHCHI et al., 1979; SASAI and IshiKAWA, 1980; Shapiro and ABDUllabeKOV, 1982; SUMITOMO and NoriTOMI, 1986).

The modelling study plays a role of connecting two main branches of tectonomagnetism: i.e., rock experiment and field work. It is also important to clarify the generating mechanism of tectonic events. STACEY (1964) gave a guideline for tectonomagnetic modelling on the basis of the reversible piezomagnetic effect. STACEY et al. (1965) presented a mechanical model of the volcanomagnetic effect. They extended the linear piezomagnetic effect based on uniaxial compression tests to the general 3-dimensional stress state. YUKUTAKE and TACHINAKA (1967) calculated the magnetic change caused by a cylindrical force source. It was the first "mechanically reasonable" model, which satisfies the elastic equation as well as the traction-free boundary condition. DAVIS (1976) calculated the piezomagnetic change due to a

*Read at 1985 IASPEI meeting, Tokyo, entitled "Some basic problems of tectonomagnetic modelling". 
hydrostatically pumped sphere, which is known as Mogl's (1958) model in volcanology. SASAI (1979) gave an analytical solution of the problem in a limiting case of infinitesimally small source sphere.

Dislocation models of earthquake faulting were studied for infinitely-long vertical strike- and dip-slip faults (SHAMSI and STACEY, 1969), a vertical strike-slip fault with semi-infinite length (TALWANI and KOVACH, 1972), a vertical rectangular strike-slip fault (JOHNSTON, 1978) and a rectangular fault having an arbitrary dip angle and slip vector (OHSHIMAN, 1980). All these dislocation problems cited above were computed numerically. SASAI (1980) showed they have analytical solutions expressed with elementary functions.

BONAFEDE and SABADINI (1980) proposed a new theoretical approach. They derived a representation theorem for the piezomagnetic field by combining the elasto-dynamic and electromagnetic equations. They showed a magnetic double layer emerges along the dislocation surface. Since Bonafede and Sabadini's theory was developed on the analogy of dielectric materials, strong interactions between the displacement and magnetic field appear explicitly in the basic equations. In the actual Earth, however, we may ignore such mutual coupling as might occur in high- $\mu$ metals. On this standpoint, SASAI (1983) reformulated the tectonomagnetic modelling procedure devised by STACEY (1964). A surface integral representation was obtained for homogeneous and isotropic elastomagnetic materials. The earthquake fault behaves as a sheet magnetic source with its intensity proportional to the seismic moment density, which is the counterpart of BONAFEDE and SABADINI's (1980) result.

In this paper we will develop a unified method to evaluate piezomagnetic field changes associated with various mechanical distortions in an elastic half-space. A wide class of tectonic models can be represented by superposing the displacement field of a unit single force operative at a point. Once the piezomagnetic potential caused by such a force is known, tectonomagnetic changes of these models can be derived quite as well from linear combinations of the prescribed potential. We may call it the fundamental piezomagnetic potential. The surface integral representation (SASAI, 1983) will be successfully applied to its derivation process. A Green's function for surface load problems is given simply as a special case of the fundamental potential. The magnetic field produced by a uniform circular load will be calculated as an application example of the present theory.

\section{Outline of the Theory}

Let us consider a simple earth model: a homogeneous and isotropic elastic half-space with a uniformly magnetized top layer. We will develop a unified treatment for calculating the piezomagnetic field associated with various types of mechanical deformation within the medium. We take the Cartesian coordinate $\left(x_{1}, x_{2}, x_{3}\right)$, in which the semi-infinite elastic medium occupies $x_{3}>0$. The magnetized region is bounded from the free surface $x_{3}=0$ to the Curie depth $x_{3}=\mathrm{H}$.

The equation of static equilibrium is given by

$$
(\lambda+\mu) \operatorname{grad} \operatorname{div} \boldsymbol{u}+\mu \nabla^{2} \boldsymbol{u}+\boldsymbol{F}=0
$$


where $\boldsymbol{u}$ and $\boldsymbol{F}$ are the displacement and the body force, while $\lambda$ and $\mu$ are Lamè's constants. The displacement field $\boldsymbol{u}$ should satisfy the traction-free boundary condition at $x_{3}=0$ :

$$
\tau_{31}=\tau_{32}=\tau_{33}=0 \quad \text { at } \quad x_{3}=0 .
$$

The stress field is related to the displacement through Hooke's law:

$$
\tau_{i j}=\lambda \delta_{i j} \operatorname{div} \boldsymbol{u}+\mu\left(\frac{\partial u_{i}}{\partial x_{j}}+\frac{\partial u_{j}}{\partial x_{i}}\right)
$$

in which $\delta_{i j}$ is the Kronecker delta.

We begin with the fundamental solution of Eq. (2.1) satisfying the condition (2.2), i.e., the displacement field caused by a single force operative at a point within an elastic half space. Let $G_{k l}$ be the $x_{k}$ component of the displacement at $Q\left(x_{1}, x_{2}, x_{3}\right)$ produced by a unit single force at $P\left(\xi_{1}, \xi_{2}, \xi_{3}\right)$ acting in the $x_{l}$ direction. We will hereafter refer to the vector representation $\boldsymbol{G}_{l}$, together with

$$
G_{k l}=G_{k l}(\boldsymbol{x} ; \xi)=G_{k l}\left(x_{1}, x_{2}, x_{3} ; \xi_{1}, \xi_{2}, \xi_{3}\right) \text {. }
$$

$\boldsymbol{G}_{l}$ satisfies the following equation:

$$
(\lambda+\mu) \operatorname{grad} \operatorname{div} \boldsymbol{G}_{l}+\mu \nabla^{2} \boldsymbol{G}_{l}+\boldsymbol{F}_{l}=0
$$

where

$$
F_{i l}=\delta_{i l} \delta\left(x_{1}-\xi_{1}\right) \delta\left(x_{2}-\xi_{2}\right) \delta\left(x_{3}-\xi_{3}\right)
$$

$\boldsymbol{G}_{l}$ fulfills the boundary condition (2.2), which is rewritten as

$$
\begin{aligned}
\mu\left(\frac{\partial G_{3 l}}{\partial x_{1}}+\frac{\partial G_{1 l}}{\partial x_{3}}\right) & =\mu\left(\frac{\partial G_{3 l}}{\partial x_{2}}+\frac{\partial G_{2 l}}{\partial x_{3}}\right) \\
& =\lambda\left(\frac{\partial G_{1 l}}{\partial x_{1}}+\frac{\partial G_{2 l}}{\partial x_{2}}+\frac{\partial G_{3 l}}{\partial x_{3}}\right)+2 \mu \frac{\partial G_{3 l}}{\partial x_{3}} \\
(l=1,2,3) & =0 \quad \text { at } \quad x_{3}=0 .
\end{aligned}
$$

Since both the basic equation and the boundary condition are linear, such a displacement field $\boldsymbol{u}$ as a linear combination of $\boldsymbol{G}_{l}$ also satisfies Eqs. (2.1) and (2.2):

$$
\boldsymbol{u}=\sum_{k} A_{k} \boldsymbol{G}_{l}\left(\boldsymbol{x} ; \xi^{(k)}\right)
$$

in which $\xi^{(k)}$ denotes the $k$-th discrete source point. This important characteristic of $\boldsymbol{G}_{l}$ can be extended to a more general class of linear combination, which is expressed by 


$$
\boldsymbol{u}=L(\xi) \boldsymbol{G}_{\boldsymbol{l}}(\boldsymbol{x} ; \xi)
$$

The functional $L(\xi)$ is called the integro-differential operator, which is defined by

$$
L(\xi) f=\sum_{\alpha}\left[\int K_{\alpha}\left(\xi, \xi^{\prime}\right) D^{\alpha} f\left(\xi^{\prime}\right) \mathrm{d} \xi^{\prime}+A_{\alpha}(\xi) D^{\alpha} f(\xi)\right]
$$

where $D$ implies the partial differential operator $\left(D=\partial / \partial \xi_{k}\right)$ and $\alpha$ is non-negative integer. Obviously the integral with respect to $\xi$ is a limiting case of the linear combination (2.8). We may also interpret derivatives with respect to $\xi$ as a kind of linear combination, in view of the fact that $\partial G_{k l} / \partial \xi_{1}$, for example, is by definition

$$
\lim _{\Delta \xi_{1} \rightarrow 0}\left\{\frac{1}{\Delta \xi_{1}} G_{k l}\left(\boldsymbol{x} ; \xi_{1}+\Delta \xi_{1}, \xi_{2}, \xi_{3}\right)-\frac{1}{\Delta \xi_{1}} G_{k l}\left(\boldsymbol{x} ; \xi_{1}, \xi_{2}, \xi_{3}\right)\right\} .
$$

Hence derivatives of $G_{k l}$ with respect to the parameter $\xi$ as well as their weighted integrals, namely the displacement field given by Eq. (2.9), are again the solution of Eq. (2.1) under the boundary condition (2.2).

Once the displacement field of a magnetoelastic body is specified, the piezomagnetic potential due to associated stresses is expressible with its displacement (STACEY, 1964; SASAI, 1983). Formally it is given by the following integral:

$$
W^{\mathrm{m}}(\boldsymbol{r})=\iiint_{V} \Delta \boldsymbol{M}^{\mathrm{m}}(\boldsymbol{u}(\boldsymbol{x})) \cdot \nabla\left(\frac{1}{\rho}\right) \mathrm{d} \boldsymbol{x}
$$

where

$$
\rho=|\boldsymbol{x}-\boldsymbol{r}|=\sqrt{\left(x_{1}-x\right)^{2}+\left(x_{2}-y\right)^{2}+\left(x_{3}-z\right)^{2}} .
$$

$\Delta \boldsymbol{M}^{\mathrm{m}}(\boldsymbol{x})$ is the stress-induced magnetization vector, whose component is given by

$$
\Delta M_{k}^{\mathrm{m}}=\beta J_{m}\left\{\frac{3}{2}\left(\frac{\partial u_{m}}{\partial x_{k}}+\frac{\partial u_{k}}{\partial x_{m}}\right)-\delta_{m k} \operatorname{div} \boldsymbol{u}\right\}
$$

$\beta$ and $J_{m}$ are the stress sensitivity and the magnetization in the $m$-th direction, respectively.

Let $U^{\mathrm{m}}{ }_{l}$ be the magnetic potential due to the stress-induced magnetization $\Delta \boldsymbol{M}^{\mathrm{m}}\left(\mathbf{G}_{\mathrm{l}}\right)$, caused by $\boldsymbol{G}_{l}$ operative to $J_{m}$ :

$$
U_{l}^{\mathrm{m}}(\boldsymbol{r} ; \xi)=\iiint_{V} \Delta \boldsymbol{M}^{\mathrm{m}}\left(\boldsymbol{G}_{l}(\boldsymbol{x} ; \xi)\right) \cdot \nabla\left(\frac{1}{\rho}\right) \mathrm{d} \boldsymbol{x} .
$$

As shown in Eq. (2.13), $\Delta \boldsymbol{M}^{\mathrm{m}}$ is given by a linear combination of $\boldsymbol{u}$. This implies the order of linear operation with respect to $\boldsymbol{x}$ and $\xi$ is allowed to exchange.

We now adopt the displacement field (2.9) and substitute it into (2.11). Interchanging the order of operations, i.e., integration with respect to $\boldsymbol{x}$ and operation of linear functional $L(\xi)$, we obtain

$$
W^{\mathrm{m}}(\boldsymbol{r})=L(\xi) U_{l}^{\mathrm{m}}(\boldsymbol{r} ; \xi)
$$


It is much easier to get at the solution $W^{\mathrm{m}}(\boldsymbol{r})$ through (2.15) than through (2.11) via (2.9). We may call $U^{\mathrm{m}}$ l the fundamental piezomagnetic potential.

Let us enumerate some problems of geophysical interest, which can be described in the form of Eq. (2.9) and hence of Eq. (2.15).

\subsection{Surface load problem}

A typical example is the dam-magnetic effect. The local magnetic change caused by surface load of a man-made lake can be calculated by integrating fundamental potentials with the weight proportional to the depth topography of the reservoir.

\subsection{Strain nuclei problem}

There are some models which explain the surface deformation of volcanoes, such as MogI's (1958) model, YoKOYAMA's (1971) model and so on. Magnetic potentials due to these models can be constructed simply by summing some derivatives of fundamental potentials.

\subsection{Dislocation problem}

A Green's function approach was proposed to estimate magnetic changes associated with any arbitrary dislocation sources in an elastic half-space (SASAI, 1980). The result was obtained by directly applying Eq. (2.11) to MARUYAMA's (1964) stress field solutions. The simplest way to derive the elementary piezomagnetic potentials is to combine some particular derivatives of $U_{l}^{\mathrm{m}}$ 's.

\subsection{Volume source problem}

The strain which is not associated with stress is called the "stress-free" strain (e.g., ESHELBY, 1957) or the "eigen" strain (MURA, 1982). Eigen strains of geophysical importance are thermal expansion and plastic deformation. Such strains behave as a sort of body force in the elastic equation. Problems containing inelastic inclusions are solved by volumetric integrals of fundamental potentials over the source region.

\section{Green's Function-Fundamental Piezomagnetic Potential}

In this section we will derive the fundamental solution of the problem, i.e., piezomagnetic potential due to the stress-induced magnetization produced by a single force operative at a point in an elastic half-space. In order to avoid confusion, we discriminate three notations of coordinates for different field quantities: the source point is represented by $\xi\left(\xi_{1}, \xi_{2}, \xi_{3}\right)$, and the displacement is a function of $\boldsymbol{x}\left(x_{1}, x_{2}, x_{3}\right)$, while the magnetic potential that of $\boldsymbol{r}(x, y, z)$. Since the magnetic field is usually measured in a free space, $z$ is assumed negative in the following calculations.

MINDLIN (1936) obtained the fundamental solution of isotropic solid of semiinfinite extent. Although Mindlin's solution is given in terms of the Galerkin vector stress function, we find an explicit form of the displacement in a textbook (e.g., MURA, 1982). We may assume a single force acts at a point $\left(0,0, \xi_{3}\right)$. Replacing $x$ and $y$ by $\left(x-\xi_{1}\right)$ and $\left(y-\xi_{2}\right)$ in the final expressions, we get the corresponding results for any arbitrary source position $P\left(\xi_{1}, \xi_{2}, \xi_{3}\right)$.

The displacement components due to a single force at $\left(0,0, \xi_{3}\right)$, which are denoted by $g_{k l}$, are given by (MURA, 1982, p. 95) 


$$
\begin{aligned}
g_{i j} & =\frac{1}{16 \pi \mu(1-v)}\left[\frac{3-4 v}{R_{1}} \delta_{i j}+\frac{1}{R_{2}} \delta_{i j}+\frac{x_{i} x_{j}}{R_{1}^{3}}\right. \\
& +(3-4 v) \frac{x_{i} x_{j}}{R_{2}^{3}}+\frac{2 x_{3} \xi_{3}}{R_{2}^{3}}\left\{\delta_{i j}-\frac{3 x_{i} x_{j}}{R_{2}^{2}}\right\} \\
& \left.+\frac{4(1-v)(1-2 v)}{R_{2}+x_{3}+\xi_{3}}\left\{\delta_{i j}-\frac{x_{i} x_{j}}{R_{2}\left(R_{2}+x_{3}+\xi_{3}\right)}\right\}\right]=g_{j i}, \\
g_{3 j} & =\frac{x_{j}}{16 \pi \mu(1-v)}\left[\frac{x_{3}-\xi_{3}}{R_{1}^{3}}+\frac{(3-4 v)\left(x_{3}-\xi_{3}\right)}{R_{2}^{3}}\right. \\
& \left.-\frac{6 x_{3} \xi_{3}\left(x_{3}+\xi_{3}\right)}{R_{2}^{5}}+\frac{4(1-v)(1-2 v)}{R_{2}\left(R_{2}+x_{3}+\xi_{3}\right)}\right], \\
g_{i 3} & =\frac{x_{i}}{16 \pi \mu(1-v)}\left[\frac{x_{3}-\xi_{3}}{R_{1}^{3}}+\frac{(3-4 v)\left(x_{3}-\xi_{3}\right)}{R_{2}^{3}}\right. \\
& +\frac{(3-4 v)\left(x_{3}+\xi_{3}\right)^{2}-2 x_{3} \xi_{3}}{\left.R_{2}^{3}+\frac{6 x_{3} \xi_{3}\left(x_{3}+\xi_{3}\right)^{2}}{R_{2}^{5}}\right],} \\
& \left.-\frac{6 x_{3} \xi_{3}\left(x_{3}+\xi_{3}\right)}{R_{2}^{5}}-\frac{4(1-v)(1-2 v)}{R_{2}\left(R_{2}+x_{3}+\xi_{3}\right)}\right], \\
g_{33} & =\frac{1,2,}{16 \pi \mu(1-v)}\left[\frac{3-4 v}{R_{1}}+\frac{8(1-v)^{2}-(3-4 v)}{R_{1}^{3}}\right. \\
& \\
& \left.=x_{3}-\xi_{3}\right)^{2} \\
& \\
&
\end{aligned}
$$

where

$$
\begin{aligned}
& R_{1}^{2}=x_{1}^{2}+x_{2}^{2}+\left(x_{3}-\xi_{3}\right)^{2} \\
& R_{2}^{2}=x_{1}^{2}+x_{2}^{2}+\left(x_{3}+\xi_{3}\right)^{2} .
\end{aligned}
$$

$v$ is Poisson's ratio.

Since the volumetric integration of Eq. (2.14) is a formidable one, we will make use of a modified formula, i.e., a surface integral representation of the tectonomagnetic field (SASAI, 1983). The piezomagnetic potential of a strained body is represented by the following formula:

$$
\begin{aligned}
W_{k}(\boldsymbol{r}) & =C_{k} \iint_{S}\left[\left\{-\frac{\partial u_{k}\left(\boldsymbol{r}^{\prime}\right)}{\partial n^{\prime}}+\frac{1}{1+v} \Delta \boldsymbol{m}_{k} \cdot \boldsymbol{n}^{\prime}\right\} \frac{1}{\rho}\right. \\
& \left.+\left\{u_{k}\left(\boldsymbol{r}^{\prime}\right)\right\} \frac{\partial}{\partial n^{\prime}}\left(\frac{1}{\rho}\right)\right] \mathrm{d} S^{\prime}
\end{aligned}
$$


where

$$
\begin{gathered}
\Delta m_{k j}=\frac{3}{2}\left(\frac{\partial u_{k}}{\partial x_{j}}+\frac{\partial u_{j}}{\partial x_{k}}\right)-\delta_{k j} \operatorname{div} \boldsymbol{u} \\
C_{k}=\frac{1}{2} \beta J_{k} \mu \frac{3 \lambda+2 \mu}{\lambda+\mu}=\beta J_{k} \mu(1+v)
\end{gathered}
$$

and $\rho$ is already given in (2.12).

In applying (3.3), any singular points of the displacement field should be excluded from the region surrounded by $S$. The source position $P(\xi)$ is the only singular point when $0<\xi_{3}<H$, while no such one is included when $\xi_{3}>H$. In either case we may subdivide the magnetized region with a few plane sheets parallel to the free surface $x_{3}=0$. Hence it is sufficient to evaluate the following integral in order to apply the formula (3.3):

$$
v_{l}^{\mathrm{m}}\left(\boldsymbol{r} ; \xi_{3} ; x_{3}\right)=C_{m} \iint_{-\infty}^{\infty}\left\{f_{m l} \frac{1}{\rho}-g_{m l} \frac{x_{3}-z}{\rho^{3}}\right\} \mathrm{d} x_{1} \mathrm{~d} x_{2}
$$

where

$$
f_{m l}=-\frac{\partial g_{m l}}{\partial x_{3}}+\frac{1}{1+v}\left\{\frac{3}{2}\left(\frac{\partial g_{m l}}{\partial x_{3}}+\frac{\partial g_{3 l}}{\partial x_{m}}\right)-\delta_{m 3} \operatorname{div} g_{l}\right\} .
$$

The outward normal $\boldsymbol{n}$ ' is tentatively defined in the downward direction (i.e. $\boldsymbol{n}^{\prime}=\boldsymbol{e}_{\mathrm{x}_{3}}$ ).

The convolution integral (3.6) can be solved by the Fourier transform method. The procedure is quite similar to that developed in previous work (SASAI, 1980), in which the Fourier transform theorem for convolution integrals and operational rules were fully utilized. All the Fourier transforms necessary for the present purpose are already given elsewhere (SASAI, 1979, 1980). Results for $v_{l}^{\mathrm{m}}$ are not shown here.

There are three typical positions of the single force source. The fundamental piezomagnetic potential can be constructed by summing contributions from two or four magnetic sheets.

Case I: when the source point lies at the free surface:

$$
U_{l}^{\mathrm{m}}=-\lim _{\varepsilon_{1} \rightarrow 0} v_{l}^{\mathrm{m}}\left(\boldsymbol{r} ; 0 ; \varepsilon_{1}\right)+v_{l}^{\mathrm{m}}(\boldsymbol{r} ; 0 ; H) .
$$

Case II: when the source is included in the magnetized region:

$$
\begin{aligned}
U_{l}^{\mathrm{m}}= & -v_{l}^{\mathrm{m}}\left(\boldsymbol{r} ; \xi_{3} ; 0\right)+\lim _{\varepsilon_{1} \rightarrow 0} v_{l}^{\mathrm{m}}\left(\boldsymbol{r} ; \xi_{3} ; \xi_{3}-\varepsilon_{1}\right) \\
& -\lim _{\varepsilon_{2} \rightarrow 0} v_{l}^{\mathrm{m}}\left(\boldsymbol{r} ; \xi_{3} ; \xi_{3}+\varepsilon_{2}\right)+v_{l}^{\mathrm{m}}\left(\boldsymbol{r} ; \xi_{3} ; H\right) .
\end{aligned}
$$

Case III: when the source lies beneath the Curie point isotherm:

$$
U^{\mathrm{m}}{ }_{l}=-v_{l}^{\mathrm{m}}\left(\boldsymbol{r} ; \xi_{3} ; 0\right)+v^{\mathrm{m}}\left(\boldsymbol{r} ; \xi_{3} ; H\right) .
$$


All these contributions are summed up and finally we obtain the fundamental piezomagnetic potential $U_{l}^{\mathrm{m}}\left(\boldsymbol{r} ; 0,0, \xi_{3}\right)$. They are summarized in the Appendix A.

\section{Surface Load Problem-Application of the Theory}

The dam-magnetic effect is an interesting subject in tectonomagnetism. The observation of local magnetic changes during the filling of a reservoir is regarded as a large-scale control experiment. Such an effect was observed at Talbingo reservoir in Australia (DAVIS and STACEY, 1972) and at Charvak reservoir in the USSR (ABDULLABEKOV et al., 1979). The model calculation of the dam-magnetic effect was conducted by DAVIS (1974). He estimated the magnetic change caused by a squareshaped load on the basis of LOVE's (1929) solution. The actual man-made lake can be approximated by the sum of squares with normal load proportional to the depth topography.

Let us apply the present theory to the surface load problem. Putting $\xi_{3}=0$ into $U^{\mathrm{m}}$ 's in the Appendix A, we obtain piezomagnetic potentials when a point force acts at the free surface. They are summarized in Appendix B. $V_{3}^{\mathrm{m}}$ is the potential caused by a normal load (i.e., the Boussinesq problem), while $V_{1}^{\mathrm{m}}$ and $V_{2}^{\mathrm{m}}$ the one by angential force along the surface (i.e., the Cerruti problem).

In Fig. 1 are shown schematically the equivalent magnetic sources of the Boussinesq problem. The horizontal magnetization $J_{x}$ produces a line of horizontal dipoles parallel to $J_{x}$ between the load point $(z=0)$ and its mirror image with respect to the Curie depth $(z=2 H)$. It also produces a quadrupole at the bottom point. The vertical magnetization $J_{z}$ results in a line of vertical dipole unti-parallel to $J_{z}$ and a minor quadrupole at the bottom. The magnetic field caused by a square load is nearly equal to that of a uniformly magnetized rectangular prism. The quadrupole term at $z=2 H$ would have negligible influence on the surface magnetic field. Now we can well understand DAVIS' (1974) results: computed piezomagnetic change are nothing but local anomaly maps of a prism-shaped magnetic mass.

Figure 1 also tells us why we always observe the decrease in the total intensity in association with the filling of a man-made lake. One may presume that considerations on the qualitative nature of the piezomagnetic effect would lead us to the same understanding; the normal load produces negative $J_{z}$ and positive $J_{x}$ component respectively. It is difficult, however, to imagine the exact feature of stress-induced magnetization owing to the complicated stress pattern near the margin of the load.

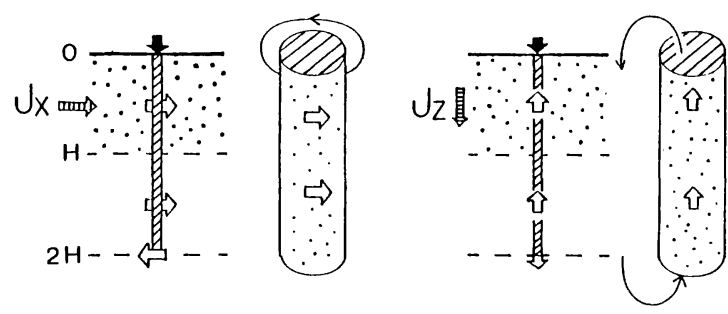

Fig. 1. A schematic representation of the equivalent magnetic sources of the Boussinesq problem: (a) Horizontal magnetization case, (b) Vertical magnetization case. 
Let us investigate here local magnetic changes caused by a uniform circular load. The main part of the solution is the field of a uniformly magnetized right circular cylinder. Unlike to the prismatic body, the solution is no longer expressible with elementary functions.

We take the $x$ axis in the magnetic north direction. The magnetization is assumed parallel to the ambient field. We replace $x$ with $x-\xi_{1}$ and $y$ with $y-\xi_{2}$ in the fundamental potentials $V_{3}^{x}$ and $V_{3}^{Z}$. We may integrate $V_{3}^{\mathrm{m}}$ over a circle of radius $a$ : $\xi_{1}^{2}+\xi_{2}^{2}=a^{2}$. With the aid of polar coordinates:

$$
x=r \cos \delta, \quad y=r \sin \delta ; \quad \xi_{1}=\xi \cos \phi, \quad \xi_{2}=\xi \sin \phi
$$

the potentials associated with the axially symmetric load $f(\xi)$ are expressed by

$$
\begin{gathered}
\Phi_{3}^{\mathrm{m}}(r, z)=\int_{0}^{a} f(\xi) \xi \mathrm{d} \xi \int_{0}^{2 \pi} V_{3}^{\mathrm{m}}(\xi, \phi ; r, z) \mathrm{d} \phi \\
(m=x, z) .
\end{gathered}
$$

We modify the inner integral with a variable transformation $\theta=1 / 2(\phi-\delta-\pi)$. After some manipulations, we obtain the following formulas:

$$
\Phi_{3}^{\mathrm{m}}=\beta J_{m} \int_{0}^{a} f(\xi) \phi_{3}^{\mathrm{m}}(\xi) \xi \mathrm{d} \xi
$$

where

$$
\begin{aligned}
\phi_{3}^{x} & =(1+v)\left[\frac{c}{\sqrt{c^{2}+(\xi+r)^{2}}}\left\{-\frac{x}{r^{2}} K(\kappa)+\frac{x}{r^{2}} \frac{\xi-r}{\xi+r} \Pi_{1}\left(-\alpha^{2}, \kappa\right)\right\}\right]_{H}^{0} \\
& -\frac{3}{2} H \frac{1}{\sqrt{c_{H}^{2}+(\xi+r)^{2}}} \frac{x}{r^{2}}\left\{\frac{c_{H}^{2}+\xi^{2}-r^{2}}{c_{H}^{2}+(\xi-r)^{2}} E\left(\kappa_{H}\right)-K\left(\kappa_{H}\right)\right\} \\
\phi_{3}^{z} & =2(2-v)\left[\frac{1}{\sqrt{c^{2}+(\xi+r)^{2}}} K(\kappa)\right]_{H}^{0} \\
& -6 H \frac{c_{H}}{\sqrt{c_{H}^{2}+(\xi+r)^{2}}} \frac{1}{c_{H}^{2}+(\xi-r)^{2}} E\left(k_{H}\right)
\end{aligned}
$$

in which $K(\kappa), E(\kappa)$ and $\Pi_{1}\left(-\alpha^{2}, \kappa\right)$ are complete elliptic integrals of the 1st, 2nd and 3rd kind. $\kappa$ and $\alpha$ are given by

$$
\begin{aligned}
\kappa_{j}^{2} & =\frac{4 \xi r}{c_{j}^{2}+(\xi+r)^{2}} \quad(j=0, H), \\
\alpha^{2} & =\frac{4 \xi r}{(\xi+r)^{2}} .
\end{aligned}
$$


$\Pi_{1}\left(-\alpha^{2}, \kappa\right)$ is defined as

$$
\Pi_{1}\left(-\alpha^{2}, \kappa\right)=\int_{0}^{\frac{\pi}{2}} \frac{d \theta}{\left(1-\alpha^{2} \sin ^{2} \theta\right) \sqrt{1-\kappa^{2} \sin ^{2} \theta}} .
$$

This can be reduced to incomplete elliptic integrals through Heuman's lambda function (MILNE-THOMSON, 1964).

The next step is to integrate Eq. (4.3). In case of the general distribution of $f(\xi)$, we have an excellent tool for numerical integrations, i.e., the double exponential formula (DEF: TAKAHASI and MORI, 1974). Values of elliptic integrals can be quickly computed with sufficient accuracy by Chebyshev approximations (e.g., HASTINGS, 1955). Heuman's lambda function is computed by NAGY's (1965) method.

As for the uniform load, we can further obtain an explicit form of (4.2). In this case, Eq (4.2) is simply expressed by

$$
\Phi_{3}^{\mathrm{m}}=W_{0} \int_{0}^{a} \xi \mathrm{d} \xi \int_{0}^{2 \pi} V_{3}^{\mathrm{m}} \mathrm{d} \phi
$$

in which $W_{0}$ is the load per unit area. There are two ways to attain the final result. One is the direct integration: we may first integrate Eq. (4.7) with respect to $\xi$ and then reduce the remaining $\phi$ integrals to elliptic ones. A similar procedure was actually conducted by NABIGHIAN (1962) to obtain the gravitational attraction of a circular cylinder. We need, however, elaborate manipulations in applying the direct method, because $V_{3}^{\mathrm{m}}$ consists of dipoles and quadrupoles with different polarizations. An alternative is the Fourier transform method by SINGH and SABINA (1978). We will follow here the latter.

Equation (4.7) can be regarded as a convolution of $V_{3}^{\mathrm{m}}$ with the following function:

$$
g(\xi)=W_{0} H(a-\xi)
$$

where $H(a-\xi)$ is the Heaviside step function. Equation (4.7) is rewritten as

$$
\Phi_{3}^{\mathrm{m}}=\iint_{-\infty}^{\infty} g\left(\xi_{1}, \xi_{2}\right) V_{3}^{\mathrm{m}}\left(x-\xi_{1}, y-\xi_{2}\right) \mathrm{d} \xi_{1} \mathrm{~d} \xi_{2} .
$$

We denote the Fourier transform of a function by an asterisk on its shoulder. We have already known the Fourier transform of $V_{3}^{\mathrm{m}}$ 's in their derivation process. Then $\Phi_{3}^{\mathrm{m}}$ is transformed as

$$
\Phi_{3}^{\mathrm{m}^{*}}=2 \pi g^{*} V_{3}^{\mathrm{m}^{*}}=2 \pi a W_{0} \frac{J_{1}(a k)}{k} V_{3}^{\mathrm{m}^{*}}
$$

The inversion of (4.10) gives rise to

$$
\Phi_{3}^{\mathrm{m}}=2 \pi a W_{0} \int_{0}^{\infty} V_{3}^{\mathrm{m}^{*}} J_{1}(a k) J_{0}(k r) \mathrm{d} k
$$

where $J_{0}$ and $J_{1}$ are Bessel functions. All the terms in $V_{3}^{\mathrm{m}^{*}}$ have a factor $e^{-c k}$ in common, 
and hence integrals in (4.11) are of the form:

$$
I[m, n ; l ; c]=\int_{0}^{\infty} J_{m}(a k) J_{n}(k r) e^{-c k} k^{l} \mathrm{~d} k
$$

in which $J_{\mathrm{m}}$ and $J_{\mathrm{n}}$ are Bessel functions of order $m$ and $n$. We can evaluate these integrals with the aid of a table by EASON et al. (1955). They are represented by complete elliptic integrals and Heuman's lambda function.

Finally we have the piezomagnetic potential caused by a uniform circular load as:

$$
\begin{aligned}
& \frac{1}{2 \pi a W_{0} \beta J_{x}} \Phi_{3}^{x}=\frac{x}{r}\left\{\frac{1+v}{2}[I(1,1 ;-1 ; c)]_{H}^{0}-\frac{3}{2} H I\left(1,1 ; 0 ; C_{H}\right)\right\} \\
& \frac{1}{2 \pi a W_{0} \beta J_{z}} \Phi_{3}^{z}=\frac{2-v}{2}[I(1,0 ;-1 ; c)]_{H}^{0}-\frac{3}{2} H I\left(1,0 ; 0 ; C_{H}\right) .
\end{aligned}
$$

In deriving $\Phi_{3}^{x}$, we can make use of the following relation:

$$
\frac{1}{2 \pi} \iint_{-\infty}^{\infty} i k_{1} f\left(k_{1}, k_{2}\right) e^{i\left(k_{1} x+k_{2} y\right)} \mathrm{d} k_{1} \mathrm{~d} k_{2}=\int_{0}^{\infty} f(k) \frac{\partial J_{0}(k r)}{\partial x} k \mathrm{~d} k
$$

The corresponding magnetic field of Eqs. (4.13) is given in the Appendix C.

In Fig. 2 are shown $\Delta X^{x}, \Delta Z^{x}, \Delta X^{z}$ and $\Delta Z^{z}$ component along the $\mathrm{N}-\mathrm{S}$ meridian. Putting $v=0.25$ and $H=15 \mathrm{~km}$, we computed field components for $a=1 \mathrm{~km}$ and the sensor height $-z=2.5 \mathrm{~m}$. Unit of the vertical axis is $\beta J W_{0}$. Numerals along the axis are given in unit of nT if we assume $\beta=2.0 \times 10^{-4} \mathrm{bar}^{-1}, J=5.0 \times 10^{-3} \mathrm{emu} / \mathrm{cc}$ and $W_{0}=10$ bar (load by $100 \mathrm{~m}$ water depth) after DAVIS (1974). The $\Delta Z^{x}$ and $\Delta X^{z}$ component become divergent at $r=a$ for $z=0$. The peak values at $r=a$ are highly dependent on the ratio $z / a$ for small $z$. At distances several times as much as $z$ from the edge, however, $\Delta Z^{x}$ and $\Delta X^{z}$ have nearly the same values for different $z / a$ 's. In other words the local edge effect rapidly diminishes as we leave the periphery of the load.

Figure 3 shows changes in the $F$ component for $W_{0}=14$ bar with the magnetic dip $I=55^{\circ}$, which are specified for the Talbingo reservoir case by DAVIS (1974). These are reasonably expected from the schematic illustration in Fig. 1. Although the shape of the load is different, numerical values are in good harmony with Davis' result for a square-shaped lake. According to these model studies, we are obliged to assume $\beta J \sim 0.1 \mathrm{nT} /$ bar in order to attain several $\mathrm{nT}$ changes against 10 bars load. This parameter is an order of magnitude larger than the ordinary one $\beta J \sim 0.01 \mathrm{nT} / \mathrm{bar}$ (e.g., $\beta=1.0 \times 10^{-4} \operatorname{bar}^{-1}, J=1.0 \times 10^{-3} \mathrm{emu} / \mathrm{cc}$ ). The observed dam-magnetic effect does exceed $5 \mathrm{nT}$ (DAVIS and STACEY, 1972; ABDUllabEKov et al., 1979). We may have to take into account tangential forces acting on the reservoir wall.

JOHNSTON et al. (1981) reported on $8 \mathrm{nT}$ increase in the geomagnetic total intensity at the catastrophic eruption of Mount St. Helens on May 18, 1980. A large-scale collapse of the summit body occurred with the eruption. The increase in the $F$ component is opposite in sign that may be anticipated from removal of magnetic material's (JOHNSTON et al., 1981). Let us estimate a possible magnetic change caused by the vanishment of load. Suppose that the collapsed mass of $2.5 \mathrm{~km}^{3}$ volume had originally a shape of a circular disk with its radius $1 \mathrm{~km}$. The normal load amounting 
(A) $X X$

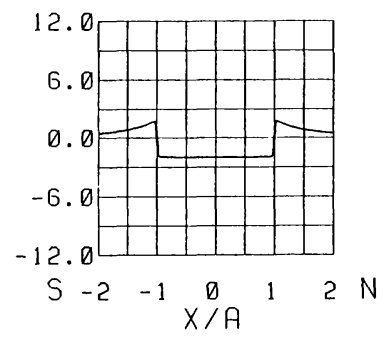

(C) $\times z$

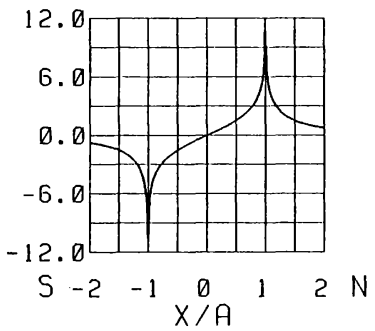

(B) $Z X$

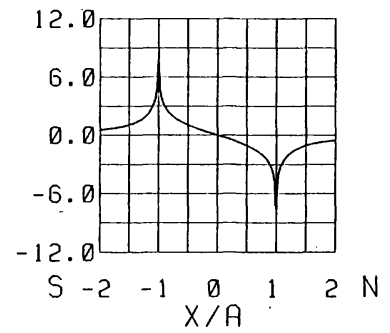

(D) $Z Z$

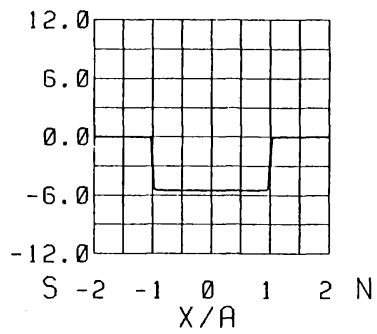

Fig. 2. Magnetic field elements due to a uniform circular load along a line passing the center from magnetic North to South: (A) $\Delta X^{x}$, (B) $\Delta Z^{x}$, (C) $\Delta X^{2}$ and (D) $\Delta Z^{z}$ component.

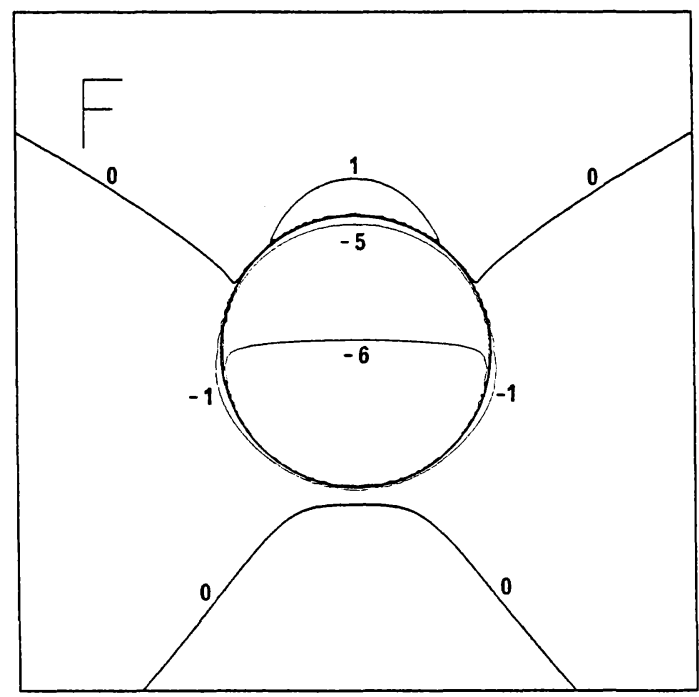

Fig. 3. The total field anomaly caused by a uniform circular load. Unit in nanoteslas. The thick centered circle indicates the location of the load. 
to 200 bars disappeared at the time of eruption if we assume the mass density 2.5. Putting $\beta J=0.1 \mathrm{nT} / \mathrm{bar}, W_{0}=-200$ bars and $I=65^{\circ}$, we obtain the magnetic change $(\Delta X, \Delta Y, \Delta Z)=(1.3,1.2,-0.5) \mathrm{nT}$ at a point $5 \mathrm{~km}$ WSW from the center of the disk: i.e. the only survived magnetometer site. The total field change is, however, merely 0.1 $\mathrm{nT}$ increase. It is difficult to explain the observation by the vanishment of the surface load. As has been claimed by JOHNSTON et al. (1981), a possible interpretation would be the internal pressure source change within the volcano.

\section{Discussion}

The volume source problem (see for example: ESHELBY, 1957; MURA, 1982) is the most interesting one among those we can apply the present method. This has not been hitherto considered in the tectonomagnetic modelling study. The thermal expansion problem is definitely important in case of the volcanomagnetic effect. We will shortly discuss here on volume source problems which we may encounter in the seismomagnetic effect.

The traditional way of tectonomagnetic modelling is the volume element method: we subdivide the magnetized crust into a number of rectangular prisms, compute average stress-induced magnetization within each element and then sum up all the contributions. The complicated stress field of dislocation models has compelled us elabolate computer work. The Green's function method overcame the difficultey. We need no longer calculate the stress-induced magnetization itself, but only specify the shape and slippage of the fault.

This simplification involves, however, a serious defect. In case of the simplest dislocation of the Volterra type, there exists stress singularity along the fault edge. The Green function method implicitly builds in such locally divergent stresses. The approximate formula of the linear piezomagnetic effect does not work at a high stress level.

This is the very point that HAO et al. (1982) criticized SASAI's (1980) result. They revived the volume element method and showed that magnetic field change diminishes for a fault model with the slip discontinuity decreasing stepwise to zero at the edge. The volume element method enables us to reproduce physically plausible magnetization changes: we may replace some approporiate values of magnetization at elements exceeding the critical stress level of the linear piezomagnetic effect. The stepwisely degrading slip model is rather too coarse to simulate the slip termination at the fault edge. We had better compute magnetic changes for a fault model with smoothly degrading dislocations by Green's function method. No stress singularity occurs for such a model (e.g., CHINNERY and PETRAK, 1967). Such improvement in computational techniques would be, however, a halfway solution in view of the essential importance involved in Hao et al.'s criticism.

Seismologists have developed the dislocation model of earthquake faulting to successfully interpret the far-field data. On the other hand, as has been predicted by model study and demonstrated by field work, measurable magnetic changes appear only near the fault. A close look at active faults tells us they consist of inhomogeneous complex in its mechanical and magnetic properties (e.g., ELECTROMAGNETIC RESEARCH GROUP FOR THE ACTIVE FAULT, 1982). The magnetic change of ordinary dislocation models comes from magnetization changes induced within the whole 
magnetic crust. Besides near the fault there should certainly exist contributions from local inelastic distortions, which might be the physical entity of earthquake faulting. In other words, magnetic data close to a fault during its entire rupture process will provide us with valuable information on the earthquake generating mechanism. The improvement in detection capability and knowledge on piezomagnetic changes associated with inelastic behaviour of rocks are the urgent subjects. The modelling study forcussing at the present target is also desirable: i.e., more realistic models of earthquakes based on fracture mechanics. The fundamental piezomagnetic potentials will be of some help to solve volume source problems which inevitably accompany these new models.

I acknowledge Professor Yukio Hagiwara for his encouragement throughout the study. I also thank Ichiro Nakagawa for his useful suggestions on the magnetic field calculation of a circular cylinder. I am indebted to an anonymous reviewer for his helpful comments.

Appendix A: Fundamental Piezomagnetic Potentials

$$
\begin{aligned}
& (l=1) \\
& \frac{8}{\beta J_{x}} U_{1}^{x}=6\left[\frac{1}{\rho}\right]_{3}^{1}-\frac{(3-4 v)(1+v)}{1-v}\left[\frac{1}{\rho+c}-\frac{x^{2}}{\rho(\rho+c)^{2}}\right]_{3}^{1} \\
& +\left(3 \frac{3-4 v}{1-v} H+\frac{1-2 v}{1-v} \xi_{3}\right)\left(\frac{1}{\rho_{3}\left(\rho_{3}+c_{3}\right)}-\frac{x^{2}\left(2 \rho_{3}+c_{3}\right)}{\rho_{3}{ }^{3}\left(\rho_{3}+c_{3}\right)^{2}}\right) \\
& -\frac{6}{1-v} H \xi_{3}\left(\frac{1}{\rho_{3}{ }^{3}}-\frac{3 x^{2}}{\rho_{3}{ }^{5}}\right) \\
& + \begin{cases}\frac{1-2 v}{1-v}\left(H-\xi_{3}\right)\left(\frac{1}{\rho_{1}\left(\rho_{1}+c_{1}\right)}-\frac{x^{2}\left(2 \rho_{1}+c_{1}\right)}{\rho_{1}^{3}\left(\rho_{1}+c_{1}\right)^{2}}\right) & \left(H<\xi_{3}\right) \\
6\left[\frac{1}{\rho}\right]_{2}^{1}-\frac{1+v}{1-v}\left[\frac{1}{\rho+c}-\frac{x^{2}}{\rho(\rho+c)^{2}}\right]_{2}^{1} & \end{cases} \\
& +\frac{3}{1-v}\left(H-\xi_{3}\right)\left(\frac{1}{\rho_{2}\left(\rho_{2}+c_{2}\right)}-\frac{x^{2}\left(2 \rho_{2}+c_{2}\right)}{\rho_{2}^{3}\left(\rho_{2}+c_{2}\right)^{2}}\right) \\
& \left(H>\xi_{3}\right), \\
& \frac{8}{\beta J_{y}} U_{1}^{y}=\frac{(3-4 v)(1+v)}{1-v}\left[\frac{x y}{\rho(\rho+c)^{2}}\right]_{3}^{1} \\
& -\left(3 \frac{3-4 v}{1-v} H+\frac{1-2 v}{1-v} \xi_{3}\right) \frac{x y\left(2 \rho_{3}+c_{3}\right)}{\rho_{3}{ }^{3}\left(\rho_{3}+c_{3}\right)^{2}}+\frac{18}{1-v} H \xi_{3} \frac{x y}{\rho_{3}{ }^{5}}
\end{aligned}
$$




$$
+ \begin{cases}-\frac{1-2 v}{1-v}\left(H-\xi_{3}\right) \frac{x y\left(2 \rho_{1}+c_{1}\right)}{\rho_{1}{ }^{3}\left(\rho_{1}+c_{1}\right)^{2}} & \left(H<\xi_{3}\right) \\ \frac{1+v}{1-v}\left[\frac{x y}{\rho(\rho+c)}\right]_{2}^{1}-\frac{3}{1-v}\left(H-\xi_{3}\right) \frac{x y\left(2 \rho_{2}+c_{2}\right)}{\rho_{2}{ }^{3}\left(\rho_{2}+c_{2}\right)^{2}} & \left(H>\xi_{3}\right)\end{cases}
$$

$$
\begin{aligned}
\frac{8}{\beta J_{z}} U_{1}^{z} & =-\frac{(5-4 v) v}{1-v}\left[\frac{x}{\rho(\rho+c)}\right]_{3}^{1} \\
& -\left(3 \frac{3-4 v}{1-v} H-\frac{1-2 v}{1-v} \xi_{3}\right) \frac{x}{\rho_{3}{ }^{3}}+\frac{18}{1-v} H \xi_{3} c_{3} \frac{x}{\rho_{3}{ }^{5}} \\
& +\left\{\begin{array}{l}
\frac{1-2 v}{1-v}\left(H-\xi_{3}\right) \frac{x}{\rho_{1}{ }^{3}} \\
-\frac{4-5 v}{1-v}\left[\frac{x}{\rho(\rho+c)}\right]_{2}^{1}-\frac{3}{1-v}\left(H-\xi_{3}\right) \frac{x}{\rho_{2}{ }^{3}}
\end{array}\right.
\end{aligned}
$$

$(l=2)$

$$
\begin{aligned}
& \frac{8}{\beta J_{x}} U_{2}^{x}=\left\{\frac{8}{\beta J_{y}} U_{1}^{y}\right\}(x \rightarrow y) \\
& \frac{8}{\beta J_{y}} U_{2}^{y}=\left\{\frac{8}{\beta J_{x}} U_{1}^{x}\right\}(x \rightarrow y) \\
& \frac{8}{\beta J_{z}} U_{2}^{z}=\left\{\frac{8}{\beta J_{z}} U_{1}^{z}\right\}(x \rightarrow y)
\end{aligned}
$$

$(l=3)$

$$
\begin{aligned}
& \frac{8}{\beta J_{x}} U_{3}^{x}=\frac{(5-4 v) v}{1-v}\left[\frac{x}{\rho(\rho+c)}\right]_{3}^{1} \\
&-\left(3 \frac{3-4 v}{1-v} H-\frac{1-2 v}{1-v} \xi_{3}\right) \frac{x}{\rho_{3}{ }^{3}}-\frac{18}{1-v} H \xi_{3} c_{3} \frac{x}{\rho_{3}{ }^{5}} \\
&+\left(\frac{1-2 v}{1-v}\left(H-\xi_{3}\right) \frac{x}{\rho_{1}{ }^{3}}\right. \\
& \frac{4-5 v}{1-v}\left[\frac{x}{\rho(\rho+c)}\right]_{2}^{1}-\frac{3}{1-v}\left(H-\xi_{3}\right) \frac{x}{\rho_{2}{ }^{3}}
\end{aligned}
$$




$$
\begin{aligned}
\frac{8}{\beta J_{y}} U_{3}^{y} & =\left\{\frac{8}{\beta J_{x}} U_{3}^{x}\right\}(x \rightarrow y) \\
\frac{8}{\beta J_{z}} U_{3}^{z} & =\frac{3-5 v+4 v^{2}}{1-v}\left[\frac{1}{\rho}\right]_{3}^{1} \\
& -\left(3 \frac{3-4 v}{1-v} H+\frac{1-2 v}{1-v} \xi_{3}\right) \frac{c_{3}}{\rho_{3}{ }^{3}}+\frac{6}{1-v} H \xi_{3}\left(\frac{1}{\rho_{3}{ }^{3}}-\frac{3 c_{3}^{2}}{\rho_{3}{ }^{5}}\right) \\
& +\left\{-\frac{1-2 v}{1-v}\left(H-\xi_{3}\right) \frac{c_{1}}{\rho_{1}{ }^{3}}\right. \\
\frac{5-7 v}{1-v}\left[\frac{1}{\rho}\right]_{2}^{1}-\frac{3}{1-v}\left(H-\xi_{3}\right) \frac{c_{2}}{\rho_{2}{ }^{3}} & \left(H<\xi_{3}\right)
\end{aligned}
$$

where

$$
[f(\rho, c)]_{k}^{j}=f\left(\rho_{j}, c_{j}\right)-f\left(\rho_{k}, c_{k}\right)
$$

and

$$
\begin{array}{lll}
\rho_{1}=\sqrt{x^{2}+y^{2}+c_{1}^{2}} \quad, & c_{1}=\xi_{3}-z \\
\rho_{2}=\sqrt{x^{2}+y^{2}+c_{2}^{2}} \quad, & c_{2}=2 H-\xi_{3}-z \\
\rho_{3}=\sqrt{x^{2}+y^{2}+c_{3}^{2}} \quad, & c_{3}=2 H+\xi_{3}-z .
\end{array}
$$

Appendix B: Green's Function for Surface Load Problem

$$
\begin{aligned}
& (l=1) \quad \begin{aligned}
\frac{8}{\beta J_{x}} V_{1}^{x} & =12\left[\frac{1}{\rho}\right]_{H}^{0}-4(1+v)\left[\frac{1}{\rho+c}-\frac{x^{2}}{\rho(\rho+c)^{2}}\right]_{H}^{0} \\
& +12 H\left\{\frac{1}{\rho_{H}\left(\rho_{H}+c_{H}\right)}-\frac{x^{2}\left(2 \rho_{H}+c_{H}\right)}{\rho_{H}{ }^{3}\left(\rho_{H}+c_{H}\right)^{2}}\right\} \\
\frac{8}{\beta J_{y}} V_{1}^{y} & =4(1+v)\left[\frac{x y}{\rho(\rho+c)^{2}}\right]_{H}^{0}-12 H \frac{x y\left(2 \rho_{H}+c_{H}\right)}{\rho_{H}{ }^{3}\left(\rho_{H}+c_{H}\right)^{2}} \\
\frac{8}{\beta J_{z}} V_{1}^{z} & =-4(1+v)\left[\frac{x}{\rho(\rho+c)}\right]_{H}^{0}-12 H \frac{x}{\rho_{H}{ }^{3}}
\end{aligned}
\end{aligned}
$$


$(l=2)$

$$
\begin{aligned}
& \frac{8}{\beta J_{x}} V_{2}^{x}=\left\{\frac{8}{\beta J_{y}} V_{1}^{y}\right\}(x \rightarrow y) \\
& \frac{8}{\beta J_{y}} V_{2}^{y}=\left\{\frac{8}{\beta J_{x}} V_{1}^{x}\right\}(x \rightarrow y) \\
& \frac{8}{\beta J_{z}} V_{2}^{z}=\left\{\frac{8}{\beta J_{z}} V_{1}^{z}\right\}(x \rightarrow y),
\end{aligned}
$$

$(l=3)$

$$
\begin{aligned}
& \frac{8}{\beta J_{x}} V_{3}^{x}=4(1+v)\left[\frac{x}{\rho(\rho+c)}\right]_{H}^{0}-12 H \frac{x}{\rho_{H}^{3}} \\
& \frac{8}{\beta J_{y}} V_{3}^{y}=\left\{\frac{8}{\beta J_{x}} V_{3}^{x}\right\}(x-y) \\
& \frac{8}{\beta J_{z}} V_{3}^{z}=4(2-v)\left[\frac{1}{\rho}\right]_{H}^{0}-12 H \frac{c_{H}}{\rho_{H}{ }^{3}},
\end{aligned}
$$

where

$$
\begin{gathered}
{[f(c)]_{H}^{0}=f\left(c_{0}\right)-f\left(c_{H}\right)} \\
\rho_{j}=\sqrt{x^{2}+y^{2}+c_{j}^{2}} \quad(j=0, H) \\
c_{0}=-z, \quad c_{H}=2 H-z .
\end{gathered}
$$

Appendix C: The Magnetic Field by the Uniform Circular Load

$$
\begin{aligned}
\frac{1}{\beta J_{x} W_{0}} \Delta X^{x}= & -\frac{1+v}{2}\left[\frac{1}{r} I_{1}(c)+\frac{x^{2}}{r^{2}}\left\{-\frac{2}{r} I_{1}(c)+I_{2}(c)\right\}\right]_{H}^{0} \\
& +\frac{3}{2} H\left\{\frac{1}{r} I_{3}\left(c_{H}\right)+\frac{x^{2}}{r^{2}}\left(-\frac{2}{r} I_{3}\left(c_{H}\right)+I_{4}\left(c_{H}\right)\right)\right\} \\
\frac{1}{\beta J_{x} W_{0}} \Delta Y^{x}= & -\frac{1+v}{2}\left[\frac{x y}{r^{2}}\left\{-\frac{2}{r} I_{1}(c)+I_{2}(c)\right\}\right]_{H}^{0} \\
& +\frac{3}{2} H\left\{\frac{x y}{r^{2}}\left(-\frac{2}{r} I_{3}\left(c_{H}\right)+I_{4}\left(c_{H}\right)\right)\right\}
\end{aligned}
$$




$$
\begin{aligned}
& \frac{1}{\beta J_{x} W_{0}} \Delta Z^{x}=-\frac{1+v}{2} \frac{x}{r}\left[I_{3}(c)\right]_{H}^{0}+\frac{3}{2} H \frac{x}{r} I_{5}\left(c_{H}\right) \\
& \frac{1}{\beta J_{z} W_{0}} \Delta X^{z}=\frac{2-v}{2} \frac{x}{r}\left[I_{3}(c)\right]_{H}^{0}-\frac{3}{2} H \frac{x}{r} I_{5}\left(c_{H}\right) \\
& \frac{1}{\beta J_{z} W_{0}} \Delta Y^{z}=\frac{2-v}{2} \frac{y}{r}\left[I_{3}(c)\right]_{H}^{0}-\frac{3}{2} H \frac{y}{r} I_{5}\left(c_{H}\right) \\
& \frac{1}{\beta J_{z} W_{0}} \Delta Z^{z}=-\frac{2-v}{2}\left[I_{2}(c)\right]_{H}^{0}+\frac{3}{2} H I_{4}\left(c_{H}\right),
\end{aligned}
$$

where

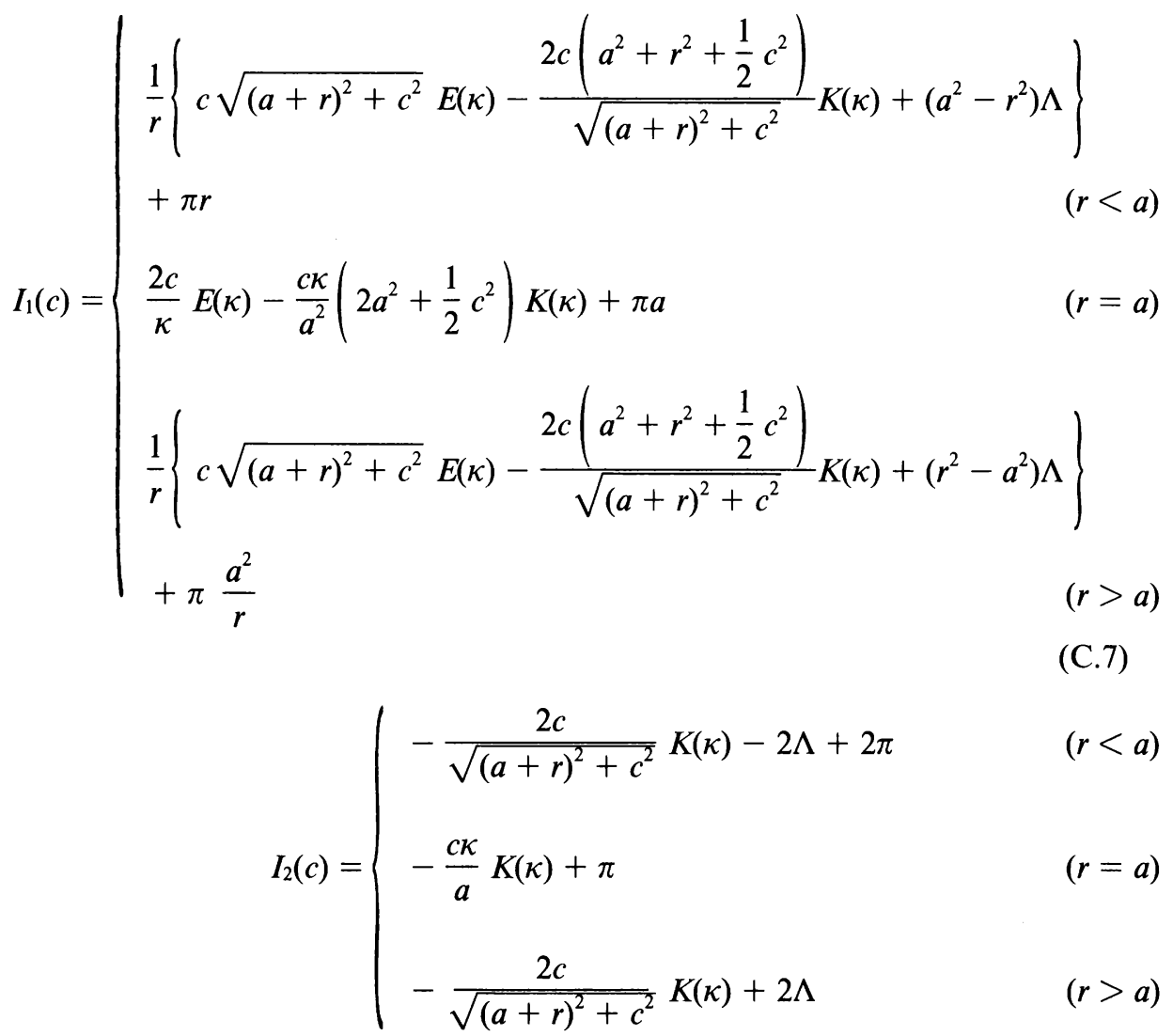

$$
\begin{aligned}
& I_{3}(c)=\frac{2 \sqrt{\left(a^{2}+r^{2}\right)+c^{2}}}{r}\left\{\left(1-\frac{1}{2} \kappa^{2}\right) K(\kappa)-E(\kappa)\right\}
\end{aligned}
$$




$$
\begin{aligned}
& I_{4}(c)=\frac{2}{\sqrt{(a+r)^{2}+c^{2}}}\left\{\frac{a^{2}-r^{2}-c^{2}}{(a-r)^{2}+c^{2}} E(\kappa)+K(\kappa)\right\} \\
& I_{5}(c)=\frac{2 c}{r \sqrt{(a+r)^{2}+c^{2}}}\left\{\frac{a^{2}+r^{2}+c^{2}}{(a-r)^{2}+c^{2}} E(\kappa)-K(\kappa)\right\},
\end{aligned}
$$

and

$$
\begin{gathered}
\Lambda=\frac{a-r}{a+r} \frac{c}{\sqrt{(a+r)^{2}+c^{2}}} \Pi_{1}\left(-\alpha^{2}, \kappa\right) \\
\alpha^{2}=\frac{4 a r}{(a+r)^{2}}, \quad \kappa^{2}=\frac{4 a r}{(a+r)^{2}+c^{2}} .
\end{gathered}
$$

Special case: at $r=0$.

$$
\begin{gathered}
\frac{1}{\beta J_{x} W_{0}} \Delta X^{x}=-\frac{1+v}{2} \pi\left[\frac{\sqrt{a^{2}+c^{2}}-c}{\sqrt{a^{2}+c^{2}}}\right]_{H}^{0}+\frac{3}{2} H \pi \frac{a^{2}}{\left(a^{2}+c_{H}^{2}\right)^{3 / 2}} \\
\Delta Y^{x}=\Delta Z^{x}=\Delta X^{x}=\Delta Y^{z}=0 \\
\frac{1}{\beta J_{z} W_{0}} \Delta Z^{z}=-(2-v) \pi\left[\frac{\sqrt{a^{2}+c^{2}}-c}{\sqrt{a^{2}+c^{2}}}\right]_{H}^{0}+3 \pi H \frac{a^{2}}{\left(a^{2}+c_{H}^{2}\right)^{3 / 2}}
\end{gathered}
$$

\section{REFERENCES}

Abdullabekov, K. N., YE. Berdaliyev, A. N. Pushkov, and V. A. Shapiro, Local variations of the geomagnetic field during the filling of a reservoir, Geomag. Aeron. (English transl.), 19, 204-206, 1979.

Bonafede, M. and R. SABadini, A theoretical approach to the seismo-magnetic effect, Boll. Geof. Teor. Appl., 22, 105-116, 1980.

Chinnery, M. A. and J. A. Petrak, The dislocation fault model with a variable discontinuity, Tectonophysics, 5, 513-529, 1967.

Davis, P. M., The piezomagnetic computation of magnetic anomalies due to ground loading by a man-made lake, Pageoph., 112, 811-818, 1974.

DAvis, P. M., The computed piezomagnetic anomaly field for Kilauea Volcano, Hawaii, J. Geomag. Geoelectr., 28, 113-122, 1976.

Davis, P. M. and F. D. StACEY, Geomagnetic anomalies caused by a man-made lake, Nature, 240, 348-349, 1972.

EASON, G., B. Noble, and I. N. SNEdDON, On certain integrals of Lipschitz-Hankel type involving products of Bessel functions, Phil. Trans. R. Soc., London, Ser. A, 247, 529-551, 1955.

Electromagnetic Research Group for the Active Fault, Low electrical resistivity along an active fault, the Yamasaki fault, J. Geomag. Geoelectr., 34, 103-127, 1982.

Eshelby, J. D., The determination of the elastic field of an ellipsoidal inclusion and related problems, Proc. R. Soc. London, Ser. A, 241, 376-396, 1957.

Hastings Jr., C., Approximations for Digital Computers, 205 pp., Princeton, 1955.

Hao, J. Q., L. M. Hastie, and F. D. STACEY, Theory of the seismomagnetic effect: a reassessment, Phys. Earth Planet. Int., 28, 129-140, 1982. 
Johnston, M. J. S., Local magnetic field observations and stress changes near a slip discontinuity on the San Andreas fault, J. Geomag. Geoelectr., 30, 607-617, 1978.

Johnston, M. J. S., R. J. Mueller, and J. Dvorak, Volcanomagnetic observations during eruptions, May-August 1980, in The 1980 eruptions of Mount St. Helens, Washington, U. S. Geol. Sur. Professional Paper, Vol. 1250, pp. 183-189, 1981.

Love, A. E. H., The stress produced in a semi-infinite solid by pressure on part of the boundary, Phil. Trans. R. Soc., London, Ser. A, 228, 377-420, 1929.

Maruyama, T., Statical elastic dislocations in an infinite and semi-infinite medium, Bull. Earthq. Res. Inst., 42, 289--368, 1964.

Milne-thomson, L. M., Elliptic integrals, in Abramowitz and Stegun (eds.), in Handbook of Mathematical Functions, pp. 587-626, Dover Publications, 1964.

Mindlin, R. D., Force at a point in the interior of a semi-infinite solid, Physics, 7, 195-202, 1936.

MoGi, K., Relations between the eruptions of various volcanoes and the deformations of the ground surfaces around them, Bull. Earthq. Res. Inst., 36, 99-134, 1958.

Mura, T., Micromechanics of Defects in Solids, 494 pp., Martinus Nijhoff Publishers, The Hague, 1982.

NABIGHIAN, M. N., The gravitational attraction of a right vertical circular cylinder at points external to it, Geofis. Pura e Appl., 53, 45-51, 1962.

Nagata, T., Tectonomagnetism, I.A.G.A. Bull., 27, 12-43, 1969.

Nagata, T., Basic magnetic properties of rocks undr mechanical stresses, Tectonophysics, 9, 167-195, 1970.

NAGY, D., The evaluation of Heuman's lambda function and its application to calculate the gravitational effect of a right circular cylinder, Pageoph., 62, 5-12, 1965.

ОhсHi, K., N. IJichi, M. Kuwashima, and M. Kawamura, Geomagnetic total force intensity variation associated with the Izu-Oshima Kinkai earthquake, 1978, Memoris Kakioka Mag. Obs., JMA, 18, 55-64, 1979 (in Japanese with English abstract).

Ohshiman, N., Local magnetic changes associated with fault activity, M. Sc. Thesis, Tokyo Inst. Tech., 1980.

RiKITAKE, T., Geomagnetism and earthquake prediction, Tectonophysics, 6, 59-68, 1968.

SASAI, Y., The piezomagnetic field associated with the Mogi model, Bull. Earthq. Res. Inst., 54, 1-29, 1979.

SASAI, Y., Application of the elasticity theory of dislocations to tectonomagnetic modelling, Bull. Earthq. Res. Inst., 55, 387-447, 1980.

SASAI, Y., A surface integral representation of the tectonomagnetic field based on the linear piezomagnetic effect, Bull. Earthq. Res. Inst., 58, 763-785, 1983.

SaSAI, Y. and Y. IshiKawa, Tectonomagnetic event preceding a $M 5.0$ earthquake in the Izu PeninsulaAseismic slip of a buried fault?, Bull. Earthq. Res. Inst., 55, 895-911, 1980.

Shamsi, S. and F. D. STACEY, Dislocation models and seismomagnetic calculations for California 1906 and Alaska 1964 earthquake, Bull. Seism. Soc. Am., 59, 1435-1448, 1969.

Shapiro, V. A. and K. N. Abdullabekov, Anomalous variations of the geomagnetic field in East Fergana-magnetic precursor of the Alay earthquake with $M=7.0$ (1978 November 21), Geophys. $J$. R. Astr. Soc., 68, 1-5, 1982.

Singh, S. K. and F. J. SABINA, Magnetic anomaly due to a vertical right circular cylinder with arbitrary polarization, Geophysics, 43, 173-178, 1978.

Smith, B. E. and M. J. S. Johnston, A tectonomagnetic effect observed before a magnitude 5.2 earthquake near Hollister, California, J. Geophys. Res., 81, 3556-3560, 1976.

StaCey, F. D., The seismomagnetic effect, Pageoph., 58, 5-22, 1964.

STACEY, F. D. and M. J. S. Johnston, Theory of the piezomagnetic effect in titanomagnetite-bearing rocks, Pageoph., 97, 146-155, 1972.

Stacey, F. D., K. G. BARr, and G. R. RobSon, The volcanomagnetic effect, Pageoph., 62, 96-104, 1965.

Sumitomo, N. and K. Noritomi, Synchronous precursors in the electrical earth resistivity and the geomagnetic field in relation to an earthquake near the Yamasaki fault, southwest Japan, J. Geomag. Geoelectr., this volume, 1986. 
Takahashi, H. and M. Mori, Double exponential formulas for numerical integration, Publ. R. I. M. S., Kyoto Univ., 9, 721-741, 1974.

Talwani, P. and R. L. Kovach, Geomagnetic observations and fault creep in California, Tectonophysics, 14, 245-256, 1972.

Yokoyama, I., A model for the crustal deformation around volcaoes, J. Phys. Earth, 19, 199-207, 1971.

YUKUTAKE, T. and H. TACHINAKA, Geomagnetic variation associated with stress change within a semiinfinite elastic earth caused by a cylindrical force source, Bull. Earthq. Res. Inst., 45, 785-798, 1967. 